\title{
Cough threshold in reflux oesophagitis: influence of acid and of laryngeal and oesophageal damage
}

\author{
L Benini, M Ferrari, C Sembenini, M Olivieri, R Micciolo, V Zuccali, G M Bulighin, \\ F Fiorino, A Ederle, V Lo Cascio, I Vantini
}

Department of Gastroenterology, Rehabilitation Hospital at Valeggio sM, University of Verona, Verona, Italy L Benini

C Sembenini

I Vantini

Laboratory of Respiratory Physiopathology, University of Verona, Italy

M Ferrari

M Olivieri

V Zuccali

V Lo Cascio

Istitute of Statistics, University of Trento, Italy

R Micciolo

Service of Digestive Endoscopy, General Hospital, Villafranca, Verona, Italy

G M Bulighin

A Ederle

ORL Istitute, University of Verona, Italy

F Fiorino

Correspondence to: Dr L Benini, Divisione di Gastroenterologia, Ospedale, 37067 Valeggio sM, Verona, Italy.

Email: vantini@

borgoroma.univr.it

Accepted for publication 21 December 1999
Abstract

Background-Gastro-oesophageal reflux is often associated with cough. Patients with reflux show an enhanced tussive response to bronchial irritants, even in the absence of respiratory symptoms.

Aim-To investigate the effect of mucosal damage (either oesophageal or laryngeal) and of oesophageal acid flooding on cough threshold in reflux patients.

Patients-We studied 21 patients with reflux oesophagitis and digestive symptoms. Respiratory diseases, smoking, and use of drugs influencing cough were considered exclusion criteria.

Methods-Patients underwent pH monitoring, manometry, digestive endoscopy, laryngoscopy, and methacholine challenge. We evaluated the cough response to inhaled capsaicin (expressed as PD5, the dose producing five coughs) before therapy, after five days of omeprazole therapy, and when oesophageal and laryngeal damage had healed.

Results-In all patients spirometry and methacholine challenge were normal. Thirteen patients had posterior laryngitis and eight complained of coughing. Twenty patients showed an enhanced cough response (basal PD5 0.92 (0.47) $\mathrm{nM}$; mean (SEM)) which improved after five and 60 days (2.87 (0.82) and $5.88(0.85) \quad \mathbf{n M}$; p<0.0001). The severity of oesophagitis did not influence PD5 variation. On the contrary, the response to treatment was significantly different in patients with and without laryngitis $(p=0.038)$. In patients with no laryngitis, the cough threshold improved after five days with no further change thereafter. In patients with laryngitis, the cough threshold improved after five days and improved further after 60 days. Proximal and distal oesophageal acid exposure did not influence PD5. Heartburn disappeared during the first five days but the decrease in cough and throat clearing were slower.

Conclusions-Patients with reflux oesophagitis have a decreased cough threshold. This is related to both laryngeal inflammation and acid flooding of the oesophagus but not to the severity of oesophagitis. Omeprazole improves not only respiratory and gastro-oesophageal symptoms but also the cough threshold. (Gut 2000;46:762-767)

Keywords: oesophagitis; cough; laryngitis; pathogenesis; treatment; gastro-oesophageal reflux
Gastro-oesophageal reflux (GOR) has been reported to be associated with a variety of respiratory disorders such as bronchial asthma, posterior laryngitis, and nocturnal apnoea. ${ }^{1-4}$ In addition, previous studies have shown an association between chronic cough and GOR. Reflux was the sole cause of the problem in $10 \%$ of patients referred for evaluation of chronic cough. ${ }^{5}{ }^{6}$ Furthermore, respiratory symptoms disappeared after effective antireflux therapy in the majority of patients with GOR. ${ }^{67}$ The mechanisms underlying this association are unclear. Two hypotheses have been suggested: (1) aspiration of refluxed gastric juices into the airways induces inflammation and cough; and (2) cough is caused by an oesophageal-tracheobronchial reflex triggered by reflux of gastric contents into the oesophagus.

The first hypothesis is supported by the frequent coexistence of GOR and laryngitis ${ }^{8}$ and by the finding of a gastro-hypopharyngeal reflux in a high proportion of patients with chronic unexplained cough. ${ }^{9}$ The stumbling block to the general acceptance of this theory has been the inability to show aspiration in most patients using radioisotope techniques. ${ }^{10}{ }^{11}$ Furthermore, individual cough episodes are better correlated with GOR events in the distal rather than the proximal oesophagus. $^{5}$

The second hypothesis is supported by the fact that in patients with chronic cough, distal oesophageal perfusion with acid increases the frequency, duration, and intensity of the cough. ${ }^{12}$ Moreover, cough is inhibited in these patients by instillation into the distal oesophagus of the local anaesthetic lignocaine. ${ }^{12}$ However, in contrast with this hypothesis is the finding that cough and reflux are not temporally related in $80 \%$ of cases and when there is a relationship, cough precedes reflux twice as often as reflux precedes cough. ${ }^{13}$ Moreover, chronic cough is rare compared with a reported $30 \%$ frequency of reflux in the general population. ${ }^{14}$ These data were obtained in patients with respiratory symptoms, the coexistence of digestive symptoms of GOR often being an exclusion criterion. ${ }^{15}$

In a previous study we found a high tussive response to inhaled capsaicin in patients with GOR but without respiratory disorders, inde-

Abbreviations used in this paper: $G O R$, gastro-oesophageal reflux; $\mathrm{FEV}_{1}$, forced expiratory volume in one second; PD5, dose of capsaicin producing five coughs; PD20, dose of methacholine causing a $20 \%$ fall in $\mathrm{FEV}_{1}$; LOS, lower oesophageal sphincter; ORL, otolaryngological. 
pendent of the actual presence of a cough. ${ }^{16}$ Hence irritant factors (capsaicin is the irritant found in red chilli peppers) might elicit coughing in GOR patients even at low concentrations (which would not cause cough in normal subjects), independent of concomitant oesophageal acidification. It is not clear which factors contribute to this lowered cough threshold. Thus the aim of this study was to investigate the role of mucosal damage (either oesophageal or laryngeal) and of acid flooding of the oesophagus on the tussive response to capsaicin in GOR patients, studied under basal conditions, after five days of treatment with omeprazole (when maximal suppression of gastric secretion was expected), and after 60 days of treatment (when the mucosa had healed).

\section{Materials and methods}

PATIENTS

We evaluated patients referred to our gastroenterological outpatient clinic for digestive symptoms suggestive of GOR. Qualifying criteria were: (1) endoscopic findings of erosive oesophagitis; (2) a pathological GOR, defined as an oesophageal acid exposure time greater than our upper normal limit (see below); (3) absence of exclusion criteria. Exclusion criteria were the presence of chronic respiratory diseases (asthma, chronic bronchitis, interstitial lung diseases) or otolaryngological (ORL) diseases (allergic rhinitis, sinusitis and postnasal drip), recent (previous two months) acute respiratory infections, smoking ( $>5$ cigarettes/ day), malignancy, pregnancy, or lactation. Patients receiving angiotensin-converting enzyme inhibitors, drugs influencing airway reactivity or lower oesophageal sphincter (LOS) tone, or gastric antisecretory treatments were also excluded.

Pulmonary and ORL diseases were excluded on the basis of a standardised interview and clinical examination. Specific investigations (pulmonary function testing, chest or sinus $x$ rays, and skin prick tests to common allergens) were performed when indicated. We evaluated 160 patients with erosive oesophagitis over a period of 24 months. Thirty eight patients met the entry criteria; the main exclusion criteria were coexistent disease (42 patients), recent antisecretory treatment (60 patients), and smoking (56 patients). Twenty one patients agreed to participate in the study and gave written informed consent.

DESIGN OF THE STUDY

Each patient underwent upper gastrointestinal tract endoscopy, stationary oesophageal manometry, 24 hour oesophageal dual probe $\mathrm{pH}$ monitoring, spirometry, methacholine and capsaicin challenges, indirect laryngoscopy, ENT examination, and quantification of digestive and respiratory symptoms of reflux.

Manometry and $\mathrm{pH}$ monitoring were performed only on entry; endoscopy and laryngoscopy were also performed at the end of the study. Symptoms were evaluated on entry, after five days of omeprazole treatment (when acid suppression was expected to be at its maximum $^{17}$ but mucosal lesions would be unaffected) and after 60 days of treatment (when oesophageal and laryngeal lesions had healed). Methacholine and capsaicin challenges were carried out at entry, and after five and 60 days. In three patients final evaluations were delayed by three weeks because testing revealed incomplete healing of oesophageal (two patients) and laryngeal lesions. Omeprazole was prescribed at a dosage of $40 \mathrm{mg}$ daily. The last dose was administered 24 hours before each testing session.

\section{OESOPHAGEAL ENDOSCOPY AND FUNCTIONAL}

TESTING

Oesophagitis was graded according to the Savary-Miller classification. ${ }^{18}$ Manometry was performed using a low compliance infusion system. LOS basal pressure and location were determined using a station pull-through technique. Peristaltic wave amplitude and speed were measured in the distal (between 10 and 5 $\mathrm{cm}$ above the LOS) and proximal (between 20 and $15 \mathrm{~cm}$ above the LOS) oesophagus. ${ }^{19}$

Proximal and distal oesophageal $\mathrm{pH}$ monitoring was performed using combined glass electrodes (Ingold Messtechnik, Germany) connected to a dual channel $\mathrm{pH}$ meter (Digitrapper Mark Gold II; Medtronic, Milan, Italy). Probes were located $5 \mathrm{~cm}$ above the LOS (distal oesophagus) and $3 \mathrm{~cm}$ below the upper oesophageal sphincter (proximal oesophagus). Patients led a normal life and completed a diary for body position, meals, reflux symptoms, and cough. The following parameters were considered: (1) oesophageal acid exposure - that is, the percentage of time with a $\mathrm{pH}$ below 4 for the distal (normal values $<4.7 \%$ ) and proximal (normal values $<0.3 \%$ ) oesophagus; (2) clearance of refluxate (mean duration of reflux episodes - that is, the mean time necessary for a decreased $\mathrm{pH}$ to return to a value above 5 for the distal (normal value < 2.6 minutes/reflux) and proximal oesophagus). Normal cut off limits were calculated as mean +2 SD, taken from two Italian multicentre studies. $^{2021}$

\section{LARYNGOSCOPY}

An experienced otolaryngologist, blind to the results of $\mathrm{pH}$ monitoring and to the patient's clinical data, performed all indirect laryngoscopies. Posterior laryngitis was scored on a 10 point (0-9) scale $^{22}$ taking into consideration mucosal integrity, mucosal surface, mucociliary function, and degree of erythema.

SPIROMETRIC TESTING AND METHACHOLINE CHALLENGE

Forced expiratory volume in one second $\left(\mathrm{FEV}_{1}\right)$ was measured using a water sealed spirometer (Biomedin, Padova, Italy). Airway reactivity to methacholine was measured using an ampoule-dosimeter device (Mefar, Brescia, Italy) as previously described. ${ }^{16}$ Patients inhaled methacholine in doses ranging from 50 to $4000 \mu \mathrm{g}$. The test ended when $\mathrm{FEV}_{1}$ decreased to more than $20 \%$ below the value measured at the beginning of the test (after inhalation of the phosphate buffer used for 
dilution of methacholine) or at a dose of $4000 \mu \mathrm{g}$. Results were expressed as the dose of methacholine causing a $20 \%$ decrease in $\mathrm{FEV}_{1}$ (PD20).

\section{CAPSAICIN CHALLENGE}

The procedure for capsaicin challenge has been described previously. ${ }^{16}$ Briefly, the capsaicin solution was prepared by dissolving $3.0 \mathrm{mg}$ of the substance (Sigma Chimica, Milano, Italy) in $10 \mathrm{ml}$ of $20 \%$ (vol $/ \mathrm{vol}$ ) ethanol in saline, obtaining a final concentration of $9.84 \times 10^{-4} \mathrm{M}$. The lowest concentrations were obtained by sequential twofold dilution of this solution. Aerosol was delivered by a jet nebuliser attached to the dosimeter, set to nebulise for 0.9 seconds at a pressure of 22 psi with an output of $10 \mu \mathrm{l} / \mathrm{breath}$. The challenge was performed by single inhalation of doses increasing from 0.076 to $4.92 \mathrm{nM}$. Ethanol in saline was given at random to increase challenge blindness. The challenge continued until five or more coughs were produced or the maximum dose of capsaicin was attained. Results were expressed as the lowest dose causing five coughs (PD5); if more than five coughs were elicited, the PD5 value was obtained by linear interpolation between the doses causing more and less than five coughs. Clearly, the lower the PD5, the greater the tussive reactivity. If five coughs were not elicited after the highest dose, PD5 was arbitrarily given the value of $9.84 \mathrm{nM}$ (that is, twice as high as the highest tested dose).

To ascertain the reproducibility of capsaicin challenge and to exclude a non-specific effect of omeprazole on cough threshold, six patients with persistent cough but with no reflux disease (five post-nasal drip, one bronchial asthma) volunteered for repeated challenges under basal conditions, and after five and 30 days of omeprazole treatment, after giving written informed consent. PD5 values did not change (median 0.15, 0.22, and $0.15 \mathrm{nM}$, respectively).

SYMPTOMS

The severity of digestive and respiratory symptoms during the week before each examination was recorded on a linear visual analogue scale from 0 (no evidence of symptoms) to 10 (intolerable severity). The following symptoms were considered: heartburn, belching, acid regurgitation, throat clearing, throat burning, cough, and hoarseness.

\section{STATISTICAL ANALYSIS}

PD5 values were transformed using base 2 logarithms before any statistical comparisons (as each time a twofold increase in capsaicin was administered). Analysis of variance for repeated measurements was used to test the difference in mean PD5 values and in mean visual analogue scores over time. The Student's $t$ test was used to evaluate the difference in mean PD 5 values between single points when analysis of variance showed the overall difference to be significant. A p value of 0.05 was always adopted. Even if significance tests were performed on log transformed PD5 values, for
Table 1 Demographic data, prevalence of symptoms and mucosal damage, and parameters of respiratory and oesophageal function in all patients

\begin{tabular}{ll}
\hline Sex $(\mathrm{M} / \mathrm{F})$ & $13 / 8$ \\
Age $(\mathrm{y})$ & $41.3(12.8)$ \\
Oesophagitis grade $(1 / 2)$ & $7 / 14$ \\
Larrngitis grade $(0 / 1 / 2 / 3)_{\mathrm{FEV}_{1}(\% \text { of predicted) }}$ & $8 / 7 / 4 / 2$ \\
$\mathrm{PD} 20(\mu \mathrm{g})$ & $11.8(2.02)$ \\
$\mathrm{LOS}$ basal tone $(\mathrm{mm} \mathrm{Hg})$ & $>4000$ (in all patients) \\
$\begin{array}{c}\text { Wave amplitude }(\mathrm{mm} \mathrm{Hg}) \\
\quad \text { (proximal/distal) }\end{array}$ & $11.5(0.32)$ \\
$\begin{array}{c}\text { Acid exposure time (\%) } \\
\quad \text { (proximal/distal) }\end{array}$ & $51.2(6.15) / 78.9(5.61)$ \\
$\begin{array}{c}\text { Oesophageal clearance (min/reflux) } \\
\quad \text { (proximal/distal) }\end{array}$ & $1.0(0.23) / 10.0(0.88)$ \\
\hline
\end{tabular}

Values are mean (SEM) or number.

the sake of clarity results are reported as means (SEM) of the original values.

\section{Results}

All 21 patients completed the study. Table 1 shows the clinical and patient data. Eight patients complained of coughing during the day and two also during the night. Thirteen patients had objective signs of slight laryngeal involvement (usually inter-arytenoideal hyperaemia). $\mathrm{FEV}_{1}$ was normal in all patients and did not change after treatment. PD20 methacholine could not be determined under basal conditions or after treatment, thus proving the absence of bronchial hyperreactivity. Neither oesophagitis grade nor the presence of laryngitis influenced these parameters.

At the beginning of the study, cough threshold was low in all except one patient (PD5 0.92 (0.46) $\mathrm{nM}$ ). There was no significant difference in PD 5 between patients with grade 1 and grade 2 oesophagitis $(0.16(0.05) v 1.29(0.68)$ $\mathrm{nM})$ or between patients with or without laryngitis $(0.6 \quad(0.37) \quad v \quad 1.43$ (1.08) $\mathrm{nM}$, respectively). There was no relationship between PD5 and the functional parameters of the proximal and distal oesophagus.

Figure 1 shows PD5 behaviour during treatment. Changes in PD5 over the whole period were highly significant $(p<0.001)$. After five days of treatment we found a significant increase in cough threshold compared with basal conditions $(p=0.001)$. Five patients did not respond to the stimulus, even at the highest

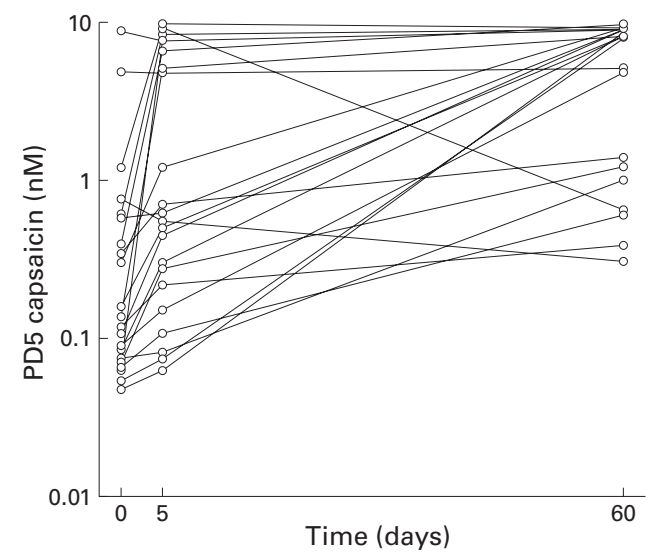

Figure 1 Results of the capsaicin challenge (expressed as the dose causing five coughs, PD5) under basal conditions and during the omeprazole treatment period (after five and 60 days). 


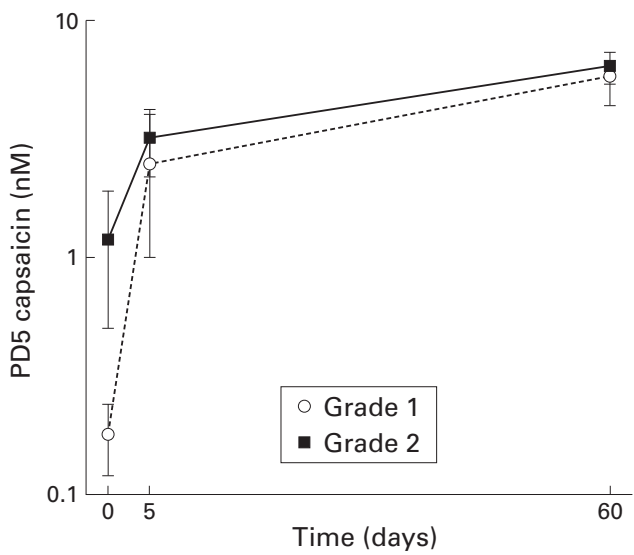

Figure 2 Results of the capsaicin challenge (expressed as the dose causing five coughs, PD5) in patients with grade 1 (seven patients) and grade 2 (14 patients) oesophagitis, under basal conditions and during the omeprazole treatment period (after five and 60 days). Values are mean (SEM) plotted on a logarithmic scale.

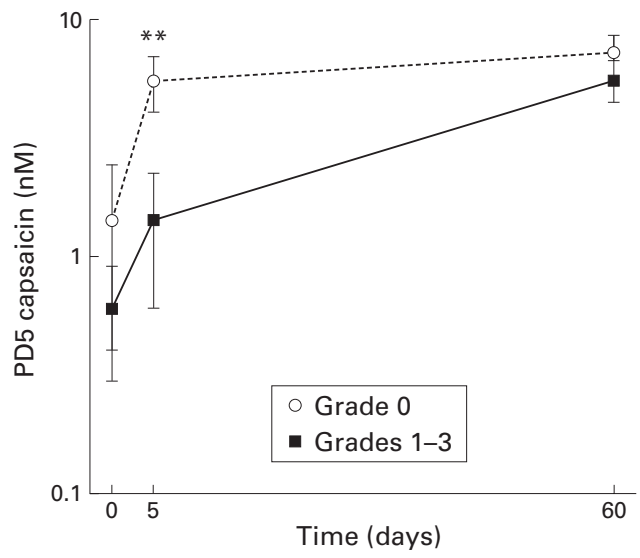

Figure 3 Results of the capsaicin challenge (expressed as the dose causing five coughs, PD5) in patients with (grades $1-3,13$ patients) and without (grade 0, eight patients) laryngitis, under basal conditions and during the omeprazole treatment period (after five and 60 days). Values are mean (SEM) plotted on a logarithmic scale. ${ }_{\star \star}^{*} p=0.003$ between groups (Student's $t$ test).

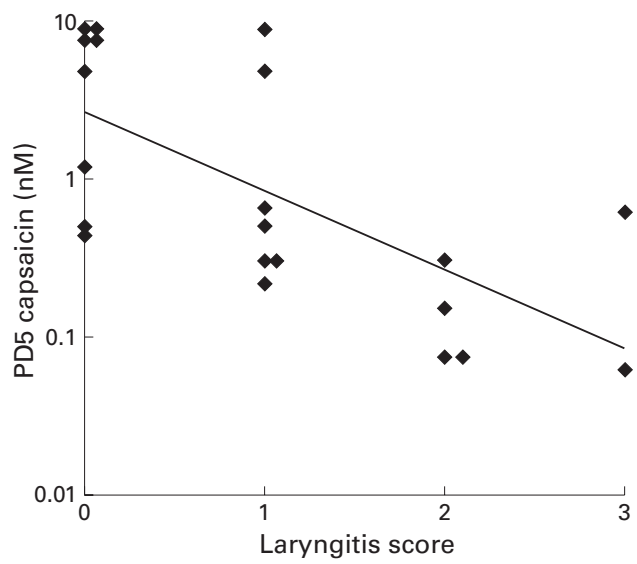

Figure 4 Relationship between grade of laryngitis and tussive response to capsaicin (expressed as the dose causing five coughs (PD5) on a log scale) after five days of omeprazole treatment. A significant negative correlation is present $(r=-0.67, p<0.001)$.

dose tested. After 60 days we found a further increase in cough threshold ( $\mathrm{p}=0.002 v$ values after five days) and seven more patients became non-responders to capsaicin. In three patients
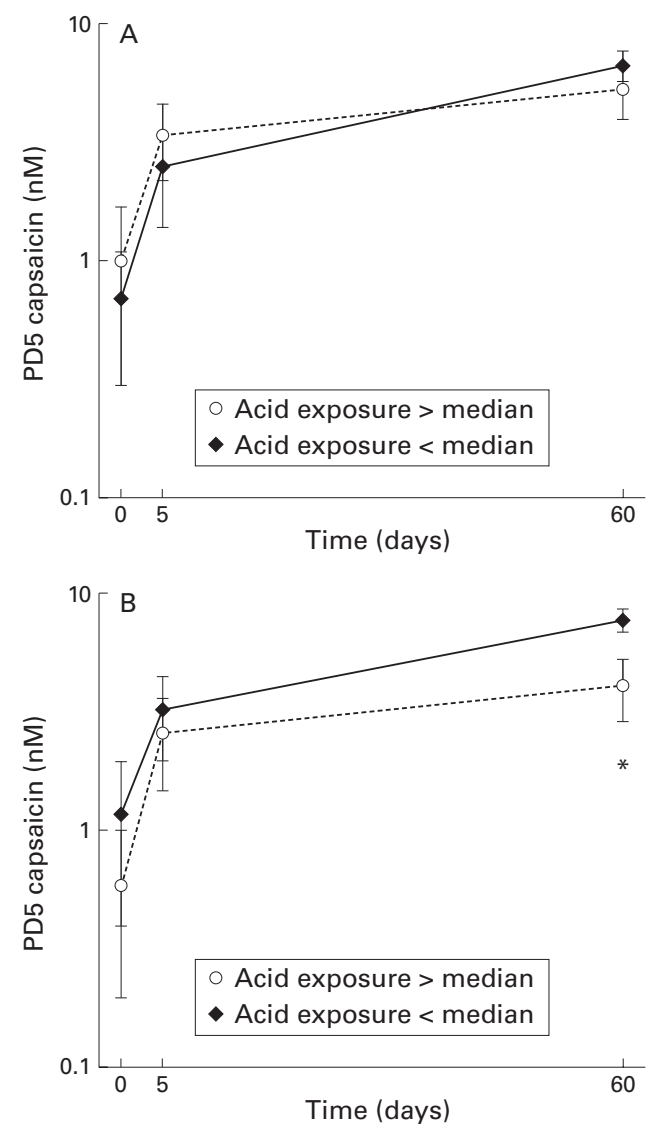

Figure 5 Variation in time of the cough threshold (expressed as the dose causing five coughs, PD5) when patients were divided according to the median values for distal $(A)$ and proximal $(B)$ oesophageal acid exposure. Patients with acid exposure greater than the median (10\% for the distal and $0.7 \%$ for the proximal oesophagus) and those with acid exposure lower than the median are shown. ${ }^{*} p<0.05$ between groups (Student's $t$ test).

PD5 did not change: in two it was already high under basal conditions $(\geqslant 4.92 \mathrm{nM}$ ).

Figure 2 shows the cough threshold in patients suffering from slight (grade 1) or more severe (grade 2) oesophagitis. There was no significant difference between the two groups under both basal conditions (as mentioned above) or during treatment.

In contrast, a significant difference $(p=0.038)$ in response to treatment was found - in patients with and without laryngitis (fig 3). In patients with no laryngitis we observed an increase in cough threshold after the first five days $(p=0.014)$ with no further increase during the next 55 days $(p=0.640)$. In patients with laryngitis, we found a small but highly significant increase after the first five days $(p=0.002)$ followed by progressive improvement with treatment $(p=0.001$ between results after five and 60 days). To explore the possibility that differences between patients with and without laryngitis may be caused by different baseline values, covariance analysis was used and baseline values were used as covariates; the results did not change.

The mean values of the two curves were significantly different after five days $(p=0.003)$ but not under basal conditions $(\mathrm{p}=0.237)$ or after 60 days $(p=0.639)$. After five days, when omeprazole had eliminated the confusing effect 
Table 2 Number of patients complaining of each symptom and mean (SEM) visual analogue score of digestive and respiratory symptoms under basal condition and during omeprazole treatment. Values refer only to patients presenting the symptom on entry. $p$ Value, significance of the overall difference (analysis of variance for repeated measurements)

\begin{tabular}{llllll}
\hline Symptom & No of patients & Baseline & 5 days & 60 days & p Value \\
\hline Belching & 20 & $7.06(0.64)$ & $3.53(0.70)$ & $1.94(0.50)$ & $<0.001$ \\
Heartburn & 15 & $5.27(0.82)$ & $0.14(0.14)$ & 0 & $<0.001$ \\
Throat clearing & 12 & $5.58(1.01)$ & $2.82(0.70)$ & $1.42(0.41)$ & $<0.001$ \\
Coughing & 8 & $5.75(1.31)$ & $2.38(0.65)$ & $0.50(0.27)$ & $<0.001$ \\
\hline
\end{tabular}

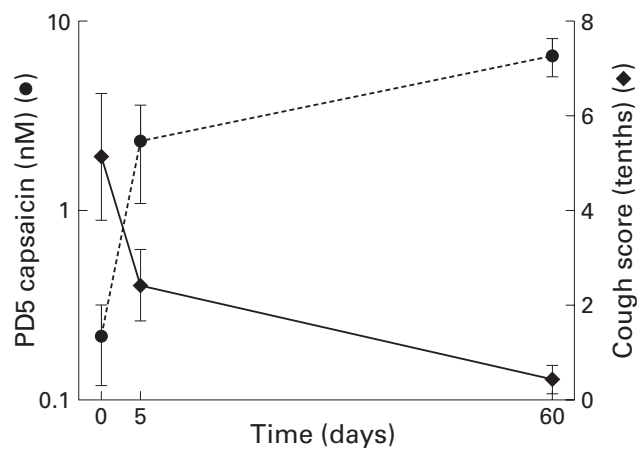

Figure 6 Relationship between the variations in the tussive response to capsaicin (expressed as the dose causing five coughs, PD5) and in coughing (expressed as tenths, visual analogue scale), only for the eight patients with cough at the beginning of the study.

of oesophageal acid exposure, a negative correlation (absent under basal conditions) between grade of laryngitis and PD5 capsaicin became apparent $(r=0.67, \mathrm{p}<0.001)$ (fig 4$)$.

Figure 5 shows the cough threshold when patients were divided according to the median values of distal and proximal oesophageal acid exposure. Neither proximal nor distal oesophageal acid exposure significantly influenced PD5 under basal conditions or during treatment.

Table 2 shows the variation in time of digestive (heartburn, belching) and respiratory (coughing, throat clearing) symptoms. Heartburn decreased rapidly and disappeared in most patients during the first five days. Cough decreased gradually and almost disappeared only after 60 days. The decrease in throat clearing was even slower. Patients with cough on entry to the study showed a tendency towards a lower cough threshold than patients without cough (PD5 0.2 (0.085) v 1.35 (0.73) $\mathrm{nM})$. However, this difference was not statistically significant $(p=0.10)$.

Figure 6 shows the relationship between variations in the tussive response to capsaicin and in coughing during treatment, only for the eight patients who had a cough at the beginning of the study. There was a clear trend for the two parameters.

\section{Discussion}

The study confirms our previous findings ${ }^{16}$ that patients with GOR have a reduced cough threshold, as demonstrated by an enhanced tussive response to capsaicin, even after exclusion of patients with some respiratory problems. A reduced cough threshold was found in the majority of our patients even if the actual prevalence of cough was lower. This finding indicates that cough is facilitated in most patients with GOR. Interaction with other fac- tors such as airway infection or pollution is necessary to induce cough, with the possible exception of inhalation in patients with particularly severe reflux. The fact that no parameters of oesophageal function or reflux discriminated between patients with or without cough lends further support to this hypothesis.

Taking into account the fact that acid was present in the oesophagus of our patients on average for only $10 \%$ of the time, it seems unlikely that acid reflux would occur during capsaicin challenge. Therefore, the actual presence of acid in the oesophagus was not the cause of the reduced cough threshold. It appears more likely that exposure of the mucosa to gastric juices produces longlasting enhancement of the cough reflex.

A low tussive threshold in GOR patients may explain the lack of association in time between acid reflux episodes and cough ${ }^{913}$ as a variety of stimuli can cause the symptom even in the absence of concomitant oesophageal acid flooding. Also, the prevalence of cough in onethird of subjects was high considering exclusion of common causes of chronic cough such as heavy smoking, post-nasal drip, bronchial asthma, and ACE inhibitor intake. This finding is probably due to the method we used to measure cough as the results of the visual analogue scale correlated with objective measurements of cough better than the questionnaires or cough diaries. ${ }^{23}$ These data confirm previous observations in the general population indicating that cough is more prevalent in subjects with GOR symptoms than in those without, ${ }^{14}$ and suggest a causal relationship between reflux disease and cough.

On entry no relationship was found between response to capsaicin and oesophagitis. The difference in PD5 between patients with grade 1 and grade 2 oesophagitis might appear at first to be of relevance. However, as the design of the study involved doubling the concentration, a wide variation in the data was expected. An overlap of $66.7 \%$ was found between the seven patients with grade 1 and the 14 patients with grade 2 oesophagitis. The variation in cough threshold during treatment was also unaffected by the grade of oesophagitis. These data suggest that the cough threshold is not influenced by the degree of mucosal inflammation and/or by exposure of subepithelial receptors. Even though our conclusions are limited by the small number of patients in the study and by the fact that all subjects had oesophagitis, this hypothesis is supported by our previous observation that oesophagitis does not play an independent role when acid exposure is taken into account. ${ }^{16}$

At first glance acid reflux and laryngitis inflammation did not correlate with cough threshold. Basal cough threshold was not influenced by proximal or distal acid exposure and was similar in patients with or without laryngitis. However, different conclusions may be drawn by studying patients after five days of treatment (that is, after effective acid suppression but before mucosal healing) when the cough threshold improved in all patients. This clearly indicates a direct role of acid exposure. 
Secondly, there was a clear effect of laryngeal inflammation. After five days the cough threshold was significantly lower in patients with laryngeal involvement and a relationship was found between laryngitis score and response to capsaicin.

The presence of laryngitis influenced the slope of the variation in cough threshold. In patients with no laryngitis the cough threshold increased after the first five days with no further change thereafter. In patients with laryngitis, the cough threshold increased after the first five days but increased even more after 60 days. In our patients laryngitis was always low grade probably because of the selection criteria. Patients with more severe laryngeal disease may require longer treatment for normalisation of mucosal inflammation and the tussive response.

These findings have practical therapeutic consequences. In patients with persistent respiratory/ORL symptoms, treatment with omeprazole for at least one month is often suggested to confirm a pathogenetic role of GOR. ${ }^{24}{ }^{25}$ Such prolonged treatment is probably necessary only in patients with posterior laryngitis; a much shorter course may be sufficient in other cases.

With regard to oesophageal acidification, neither proximal nor distal exposure to gastric juices significantly affected the cough threshold under basal conditions or during treatment (variance analysis). However, more severe proximal exposure seems to cause a slower and incomplete normalisation of the tussive response (fig 5) with a significant difference in PD5 values after 60 days $(\mathrm{p}<0.05$, Student's $t$ test). We are aware that a $t$ test should not be performed when the result of the variance analysis is not significant but the test for interaction is likely to have a low power. Thus differences in the pattern of the tussive response according to the degree of proximal acidification cannot be ruled out.

Twenty four hour $\mathrm{pH}$ monitoring was not repeated at the end of treatment and we cannot exclude the fact that persistent acid reflux may have caused incomplete normalisation of the cough threshold in some patients. However, healing of oesophagitis and the rapid disappearance of heartburn in all patients after treatment argue against this hypothesis.

Our study was not designed to specifically investigate the effect of omeprazole on respiratory and digestive symptoms but a few observations were apparent. Firstly, acid related symptoms such as heartburn disappeared after the first days of treatment. In contrast, respiratory symptoms decreased more slowly and in some patients they were still present at the end of treatment confirming their relationship with mucosal inflammation. Interestingly, the decrease of spontaneous cough, as assessed by the visual analogue scale, was similar to the change in tussive response to capsaicin. This finding indicates that the capsaicin challenge is a reliable index of the variation in time of coughing.
In conclusion, our study shows that patients with reflux oesophagitis have a reduced cough threshold even in the absence of cough. The reduced cough threshold is related to both laryngeal inflammation and acid flooding of the oesophagus. Oesophagitis plays a marginal, if any, role. Omeprazole treatment improves not only respiratory and gastro-oesophageal symptoms but also the cough threshold. This reduced cough threshold may represent the pathogenetic mechanism of unexplained chronic cough in some patients with gastrooesophageal reflux.

1 Overholt RH, Vorhees RJ. Oesophageal reflux as a trigger in asthma. Dis Chest 1966;49:464-6.

2 Mays EE. Intrinsic asthma in adults: association with gastroesophageal reflux. $\mathcal{F A M A}$ 1976;236:2626-8.

3 Kambic V, Radsel Z. Acid posterior laryngitis, aethiology, histology, diagnosis and treatment. $\mathcal{f}$ Laryngol Otol 1984;98:1237-40.

4 MacFadyen UM, Handry GMA, Simpson H. Gastroesophageal reflux in near-miss sudden infant death 1983;58:87-91.

5 Irwin RS, Zawacki JK, Curley FJ, et al. Chronic cough as the sole presenting manifestation of gastroesophageal reflux. sole presenting manifestation of gastroes

6 Irwin RS, Curley FJ, French CL. Chronic cough: the specIrwin RS, Curley FJ, French CL. Chronic cough: the spec-
trum and frequency of causes, key components of the diagnostic evaluation, and outcome of specific therapy. Am Rev Respir Dis 1990;141:640-7.

7 Ing AJ, Ngu MC, Breslin ABX. A randomised double blind placebo controlled cross-over study of ranitidine in patients with chronic persistent cough associated with gastroesophageal reflux. Am Rev Respir Dis 1992;145(suppl):A11.

8 Jakob P, Kharilas PJ, Herzon G. Proximal oesophageal $\mathrm{pH}$-metry in patients with reflux laryngitis. Gastroenterology 1991;100:305-10

9 Paterson WG, Murat BW. Combined ambulatory oesophageal manometry and dual-probe $\mathrm{pH}$-metry in evaluation of patients with chronic unexplained cough. Dig Dis Sci 1994; 39:1117-25.

10 Ghaed N, Stein MR. Assessment of a technique for scintigraphic monitoring of pulmonary aspiration of gastric contents in asthmatic with gastroesophageal reflux. Ann contents in asthmatic

11 Crausaz FM, Favez G. Aspiration of solid food particles into lungs of patients with gastrooesophageal reflux and chronic bronchial disease. Chest 1988;93:376-8.

12 Ing AJ, Ngu MC, Breslin ABX. Pathogenesis of chronic persistent cough associated with gastro-esophageal reflux. $\mathrm{Am}$ f Respir Crit Care Med 1994;149:160-7.

13 Laukka MA, Cameron AJ, Schei AJ. Gastroesophageal reflux and chronic cough: which comes first? F Clin Gastroenterol 1994;19:100-4.

14 Ruth M, Månsson I, Sandberg N. The prevalence of symptoms suggestive of oesophageal disorders. Scand f Gastroenterol 1991;26:73-81.

15 Ing AJ, Ngu MC, Breslin ABX. Chronic persistent cough and gastroesophageal reflux. Thorax 1991;46:479-83.

16 Ferrari M, Olivieri M, Sembenini C, et al. Tussive effect of capsaicin in patients with gastroesophageal reflux without cough. Am F Respir Crit Care Med 1995;151:557-61.

17 Olbe L, Cederberg C, Lind T, et al. Effect of omeprazole on gastric acid secretion and plasma gastrin in man. Scand $\mathcal{f}$ Gastroenterol 1989;24(suppl 166):27-32.

18 Savary M, Miller G. The oesophagus. Solothurn, Switzerland: Verlag Gassman, 1978:135-42.

19 Howard PJ, Benini L, Pryde A, et al. Contribution of lower oesophageal sphincter pressure and relaxation in diagnostic oesophageal manometry. Eur $\mathcal{f}$ Gastroenterol Hepatol 1990; 2:37-44.

20 Baldi F, Ferrarini F, Longanesi AM, et al. Ambulatory 24-hour oesophageal $\mathrm{pH}$ monitoring in normal subjects: a multicentre study in Italy. Ital f Gastroenterol 1991;23:477multice.

21 Baldi F, Bancaccio ML, Cappiello R, et al. Proximal G-E reflux in patients with unexplained otolaryngologic (ORL) symptoms. A multicenter study in Italy. Gastroenterology 1997;112:A63.

22 Kamel PL, Hanson D, Kahrilas PJ. Omeprazole for the treatment of posterior laryngitis. Am f Med 1994;96:321-6.

23 Myers JD, Shakur BHK, Ind PW, et al. Use of ambulatory cough recording to validate other measures of cough. Eur Respir f 1997:(suppl):422S.

24 American Gastroenterological Association. American Gastroenterological Association medical position statement: guidelines on the use of oesophageal $\mathrm{pH}$ recording. Gastroenterology 1996;110:1981-96.

25 Irwin RS, French CL, Curley FJ, et al. Chronic cough due to gastroesophageal reflux. Clinical, diagnostic, and pathogegastroesophageal reflux. Clinical, diagn
netic aspects. Chest 1993;104:1321-2 